\title{
Cavity-soliton motion in the presence of device defects
}

\author{
E. Caboche, S. Barland, M. Giudici, and J. Tredicce \\ Institut Non-Linéaire de Nice, UMR 6618, Centre National de la Recherche Scientifique/Université de Nice Sophia-Antipolis, \\ 06560 Valbonne, France
}

G. Tissoni and L. A. Lugiato

CNISM and Dipartimento di Fisica e Matematica, Università dell'Insubria, Via Valleggio 11, 22100 Como, Italy

(Received 9 September 2009; published 10 November 2009)

\begin{abstract}
Cavity solitons (CSs) are localized structures appearing as single intensity peaks in the homogeneous background of the field emitted by a nonlinear (micro) resonator driven by a coherent field (holding beam). By introducing a phase gradient in the holding beam, it is possible to induce CS drift. This motion is strongly influenced by the presence of defects in the device structure. We analyze numerically two situations that appeared in the experiments. In the first one, a structure is self-generated on the defect and a regular sequence of moving CS originates from it. We investigate the properties of this "tap" of CS as a function of the defect characteristics and of the parameters values. The second situation corresponds to the interaction between a moving CS and a defect, which plays a fundamental role in CS applications such as the delay line or the shift register.
\end{abstract}

DOI: 10.1103/PhysRevA.80.053814

PACS number(s): 42.65.Tg, 42.65.Sf, 42.79.Ta

\section{INTRODUCTION}

Spatial and temporal localization of light is one of the hottest topics in non linear optics [1-3]. Solitons (both spatial and temporal) have been largely investigated in the framework of propagative systems. In the last decade, a novel approach to this problem was developed borrowing concepts from pattern formation in dissipative systems [4,5]. In particular, localized structures, i.e., a cellular part of an extended pattern, appeared as a new promising solution to reach light localization [6,7]. Beyond the transverse localization, localized structures own properties that make them very attractive for applications: they can be independently switched on and off by a local optical perturbation [8] and they can be moved and positioned by applying a parameter gradient into the system [9]. Single-peak localized structures have been also called cavity solitons (CSs) [5]. In semiconductor devices, they have been predicted $[10,11]$ and experimentally observed [12] in a broad-area (more than $150 \mu \mathrm{m}$ diameter) vertical cavity surface emitting laser (VCSEL) driven by injection of a coherent and homogeneous field (holding beam) and biased above transparency but below threshold (amplifying regime). Typical transverse size of CS is around $10 \mu \mathrm{m}$ and they appear in a broad region of the parameter space upon proper tuning of VCSEL bias, holding beam amplitude and detuning between the cavity resonance and the injected field frequency [13]. Individual addressing has been shown experimentally by injecting into the cavity a writing beam. This is a narrow beam, having approximately of the same size of CS and coherent with the holding beam [14]. CS motion has been demonstrated upon introduction of a phase gradient in the holding beam, paving the way to possible application of CS to delay line and shift-register memory [15]. CS array reconfiguration has been shown by introducing a phase landscape in the holding beam profile through a spatial light modulator. Independently of their initial position, CS migrates toward the phase maxima and their final arrangement reproduces the phase landscape inserted
[16]. Despite these successful implementations of CS properties, we are still far from a full implementation of their potential in nonprototype devices. A difficulty comes from the engineering of broad-area (larger than $150 \mu \mathrm{m}$ diameter) VCSELs. State-of-the-art fabrication process does not fully prevent from formation of small size "defects" (diameter ranging from few $\mu \mathrm{m}$ up to few tens of $\mu \mathrm{m}$ ) in the transverse plane of the laser. These are in general local spatial variations of the semiconductor resonator characteristics (thickness, electrical and optical properties, optical gain) $[17,18]$. Local defects affect CS addressing inducing spontaneous formation of CS at undesired positions and they prevent from an arbitrary addressing of CS through the whole transverse section of the device [13]. Local defects also affect CS positioning since they may trap, annihilate, or deviate the moving CS. This fact strongly limits the possibility of controlled movement of CS on long distance and the possibility of arbitrary reconfiguration of CS positions $[15,16]$. The influence of defects on the CS trajectory was used in order to map the defect positions in a VCSEL device. The results obtained have shown an extremely rugged surface for the devices available [19]. Finally, in a recent paper, it was shown that the interplay between these defects and a phase gradient in the holding beam may lead to a regular sequence of CSs originating from the defect and moving in the gradient direction [20], a sort of CS “tap."

In this paper, we analyze numerically in details how defect characteristics influence the CS spatiotemporal dynamics in presence of a phase gradient in the holding beam. In particular, we will show that, depending on defect depth and steepness, different dynamical behaviors can be obtained. In the next section, we will describe how defects are introduced in the semiconductor cavity model and how a holding beam phase gradient affects the CS speed and their stability. In Sec. III, we will report on different spatiotemporal dynamics of CS as a function of defects characteristics and phase gradient strength in the CS tap. In Sec. IV, we will analyze a different situation, where a traveling CS meets a defect 


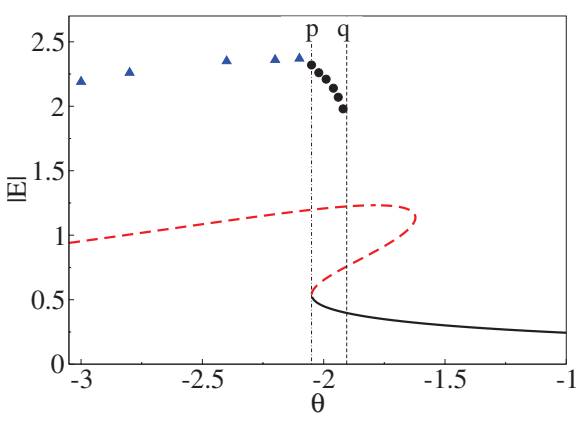

FIG. 1. (Color online) Stationary solutions for a homogeneous device as a function of the cavity detuning parameter $\theta$. Solid (dashed) line represents the stable (unstable) homogeneous steady state. Circles (triangles) represent the maximum intensity of stable CSs (patterns). Other parameters are $I=2.0024$ and $E_{I}=0.792$. The region between the two lines $p$ and $q$ is the stability region of CSs corresponding to $-2.06 \leq \theta \leq-1.905$.

placed on its trajectory. In Sec. V, we will discuss the relation between the dynamical behaviors described above and CS applications. Finally, in Sec. VI, we will draw our conclusions.

\section{THEORETICAL MODEL}

Our model describes a broad-area VCSEL, biased below threshold, in the paraxial and mean-field limit approximations and it reads [11]

$$
\frac{\partial E}{\partial t}=-\left[(1+i \theta) E-2 C(1-i \alpha)(N-1) E+-E_{I}-i \nabla_{\perp}^{2} E\right],
$$

$$
\frac{\partial N}{\partial t}=-\gamma\left[N+(N-1)|E|^{2}-I-d \nabla_{\perp}^{2} N\right],
$$

where $E$ is the normalized slowly varying envelope of the electric field and $N$ is the carrier density, $\gamma$ is the ratio between the nonradiative recombination rate $\gamma_{\|}$and the cavity decay rate $\kappa\left(\gamma=\gamma_{\|} / \kappa\right)$, and $\theta$ is the cavity detuning parameter $\theta=\left(\omega_{c}-\omega_{0}\right) / \kappa$, with $\omega_{0}$ being the injected field frequency and $\omega_{c}$ the cavity resonance frequency. $E_{I}$ is the normalized input field, $I$ is the normalized injected current, $2 C$ is the bistability parameter, $\alpha$ is the linewidth enhancement factor, $\nabla_{\perp}^{2}$ is the transverse Laplacian, and $d$ is the carrier diffusion coefficient.

Time is scaled to the photon lifetime $\kappa^{-1}$ and the spatial variables $x$ and $y$ are scaled to the square root of the diffraction parameter $a$; for this kind of cavities, we can assume a time unit of about $10 \mathrm{ps}$ and a space unit of about $4.5 \mu \mathrm{m}$ [11]. In the numerical simulations performed in this paper, the following parameters have been fixed: $C=0.45, \alpha=5, \gamma$ $=0.01$, and $d=0.052$. Our control parameters are then $\theta, E_{I}$, and $I$.

The S-shaped input-output curve for the homogeneous stationary solution of Eqs. (1) and (2), showing the intracavity field amplitude as a function of $\theta$, is shown in Fig. 1 for a homogeneous device. The broken part of the curve shows the unstable region where, due to a modulational instability, the system generates a spatially modulated solution. CSs coexist with the stable low-intensity homogeneous solution for $-2.06 \leq \theta \leq-1.905$ (that is, between the two dashed line s $p$ and $q$ ), while for $\theta<-2.06$, only patterns exist and for $\theta>-1.905$, only the lower homogeneous solution is stable.

\section{A. Introduction of device defects in the model}

In this paper, we focus our analysis on small-scale defects that appear in the device transverse section. We call defect any local variation of a structural parameter of the device on a scale ranging from a few $\mu \mathrm{m}$ to a few tens of $\mu \mathrm{m}$, i.e., having a size comparable to the CS size. These inhomogeneities are typically generated during the device growth or post-processing stages and they have been experimentally analyzed $[17,18]$. Several structural parameters can be involved: the resonator length (Bragg reflectors layers thickness variations and interface roughness), the optical gain, and the refractive index (transverse inhomogeneity of doping level). These defects appear to be unavoidable at the stateof-the-art of the laser manufacturing and they affect the translational invariance of the VCSEL device usually assumed for the generation of CS. As a consequence, some spots in the transverse section of the device may display different parameter values with respect the rest of the device; in particular, the parameter values may be incompatible with CS existence, even if the largest part of the device is set for CS suitable parameter values. In these conditions, CS addressing becomes impossible at certain points of the device.

Moreover, it is well known that any spatial variation of the parameters induces a drift of the CS $[9,21]$. Hence, smallscale defects may behave as attractive or repulsive regions for CS thus imposing strong constraints on their positions. In recent papers, the effects of small-scale inhomogeneities on CS movement have been analyzed [19,22]. In [19], CSs have been dragged by applying moving fringes in the holding beam acting as an external force on CS. The direction of this force has been varied and the resulting CS trajectories have been analyzed. Because of the attractive or repulsive effects of defects, the CS trajectories deviate from the directions imposed by the external force. Accordingly, it has been possible to construct a map of defects for the specific device used. The same experiment has been performed numerically in order to verify the role of defect versus the dragging force. The broad-area injected VCSEL is described using the rate equations (1) and (2). Device defects in the transverse plane of the device are simulated by introducing a local spatial dependence in the value of the cavity detuning parameter. In physical terms, this means to identify the defects as local variations of the resonator thickness (due to Bragg reflectors roughness). This approach has been successfully used in order to reproduce numerically the device defect map observed experimentally, thus validating the description of defects in terms of the parameter $\theta$. The same assumption has been made in Ref. [20], leading to the numerical confirmation of the periodic regime observed in the experiment (the CS tap).

In this paper, we build on this result in order to analyze in details the role of defect characteristics on CS dynamics. 


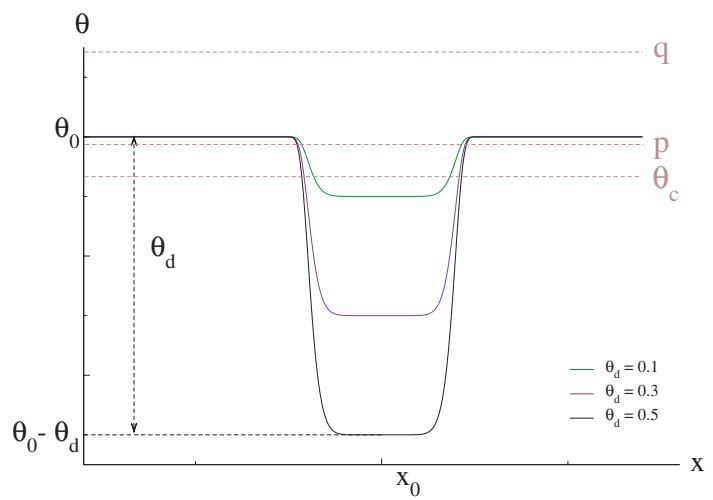

FIG. 2. (Color online) Spatial profile (cut at $y=y_{0}$ ) of the superGaussian attractive defect described by Eq. (3) for different value of $\theta_{d}$. Other defect characteristics are $\beta=3, \theta_{0}=-2.0472$, and $\sigma_{d}$ $=11.25 \mu \mathrm{m}$. Horizontal lines are plotted for reference and they indicate the critical values of $\theta$ as described in the text.

Then, a defect is described by introducing a super-Gaussian spatial profile of $\theta$,

$$
\theta(x, y)=\theta_{0}-\theta_{d} \exp \left[-\left(\frac{\left(x-x_{0}\right)^{2}+\left(y-y_{0}\right)^{2}}{\sigma_{d}^{2}}\right)^{\beta}\right],
$$

where $\theta_{0}$ is chosen in the range where CSs are stable $\left(p<\theta_{0}<q\right)$ and $\beta=3$ unless differently stated. According to the experimental observations $[17,18]$, the defect size is assumed comparable to the CS size. Unless differently stated, we assume $\sigma_{d}=11.25 \mu \mathrm{m}$ which means a defect diameter at half depth (height) of $21.2 \mu \mathrm{m}$.

If $\theta_{d}$ is positive, it corresponds to the defect depth and we define the defect as attractive. On the contrary, for a repulsive defect, $\theta_{d}$ is negative and it corresponds to the defect height. In Fig. 2, we plot the attractive defect profile for different values of the defect depth $\theta_{d}$. It is evident that a change of the depth implies also a change of the steepness of the defect walls, i.e., of the gradient of $\theta$.

We performed numerical simulations of Eqs. (1) and (2). We used a split-step method with fast Fourier transform (FFT) to integrate the transverse Laplacian terms on a spatial grid of $128 \times 128$ points with periodic boundary conditions. The finite size of the device has been simulated by assuming an injected current with circular profile (diameter of about $200 \mu \mathrm{m})$.

In the case of an attractive defect, we have observed that, if the defect depth is sufficiently large, a high-intensity structure spontaneously forms upon the defect. This happens because only a "pattern" solution is stable there. The same bifurcation occurs in the homogeneous device shown in Fig. 1 when $\theta<p$ and patterns develop throughout the entire device section. Actually, the single-peaked structure generated on the defect can be intuitively viewed as a "portion" of a pattern, the characteristics of which are strongly dependent on the defect size and shape. In particular, it is important to point out that the bifurcation value of $\theta$ leading to the structure formation in the defect $\left(\theta_{c}\right)$ is slightly different (more negative) from the bifurcation value $p$ calculated in the homogeneous case and it depends on the defect characteristics.

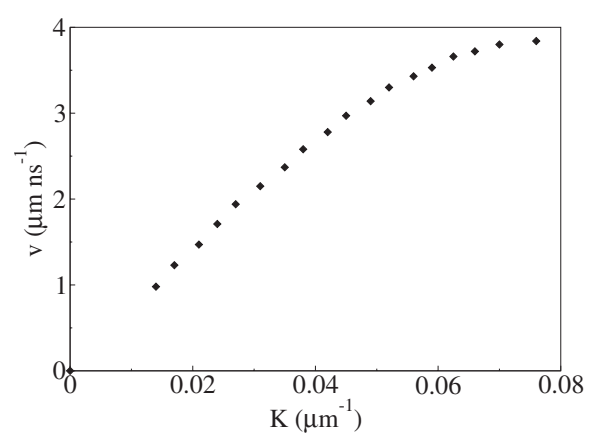

FIG. 3. CS drift speed vs phase gradient in the holding beam. Other parameters are as in Fig. 1.

For the defect size considered in Fig. 2, the single-peaked structure forms spontaneously for $\theta_{0}-\theta_{d}<\theta_{c}=-2.114$ (see Fig. 8). If $\sigma_{d}$ is increased, $\theta_{c}$ converges to $p$, but over a critical defect size an extended pattern forms as expected.

The structure generated on the attractive defect is also pinned by the defect because of the force engendered by the gradient of $\theta$ at the defect walls. The pinning force generated by the defect will be analyzed in Sec. IV.

In the case of a repulsive defect, if the defect height is sufficiently large, no CS can live or be addressed upon the defect. This happens because only the low-intensity "homogeneous" solution is stable there. The same bifurcation occurs in the homogeneous device shown in Fig. 1 when $\theta$ $>q$ and homogeneous solution is the only possible solution throughout the entire device section. As a matter of fact, if a writing beam is used targeting the defect, the system locally relaxes to the low-intensity solution as soon as the perturbation is removed. At the same time, the gradient of $\theta(x, y)$ at the defect walls generates a repulsive force for CS brought close to the defect, as it will be analyzed in Sec. IV.

\section{B. Introduction of the phase gradient in the holding beam}

As already mentioned, CSs are known to couple to any spatial variation of a system parameter, which represents a perturbation of the translational symmetry: they will therefore drift transversely on any parameter gradient with a velocity proportional to the gradient amplitude, at least in the perturbative limit [21]. From the experimental point of view, a gradient can be introduced in the holding beam phase by piezoelectrically tilting the mirror injecting the holding beam in the VCSEL cavity [15]. Moreover, the global phase of the holding beam is not a critical parameter for the CS stability and this simplifies the introduction of the gradient, which should therefore preserve CS existence conditions. For all these reasons, CS drift is preferably generated by introducing a gradient in the holding beam phase and, in this paper, we will limit the analysis to this situation. In the models (1) and (2), a linear gradient is introduced in the holding beam phase by putting $E_{I}=E_{I 0} \exp (i \vec{K} \cdot \vec{x})$. The effect of a phase gradient in the holding beam on CS speed has been analyzed numerically in [15]. For the parameters we used, the CS speed $v$ as a function of $K$ is plotted in Fig. 3. $v$ depends linearly on $K$ on a large range, where the perturbative limit is still valid, 


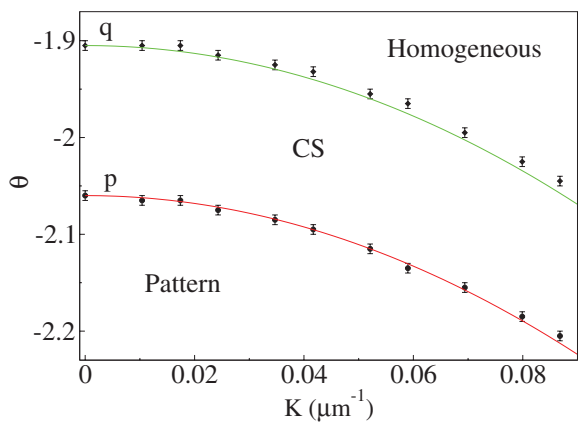

FIG. 4. (Color online) CS existence domain as a function of $\theta$ and $K$ in a homogeneous device. Circles correspond to the critical values obtained by numerical integration of Eqs. (1) and (2). Lines correspond to the correction of $\theta$ as a function of $K$ according to Eq. (4) applied to the values of $p$ and $q$, delimiting the CS existence domain when $K=0$. Other parameters are as in Fig. 1.

and then it saturates. For higher values of $K$, the moving CS solution is not stable anymore.

Since the global holding beam phase is not a critical parameter for the CS existence, it is surprising that, for large values $K, \mathrm{CS}$ stability is affected. A strong clue to understand this effect can be found in $[9,23]$. In these papers, the problem of drifting CS in an injected cavity is treated analytically using a single equation for the electromagnetic field propagating in an instantaneous two-level passive medium. In particular, it has been shown that the addition of a linear phase gradient in the holding beam generates, after a suitable change of variables, two extra terms in the field equation. The first one transforms the time derivative of the field into a convective derivative, thus indicating that traveling CSs are solutions of the field equation. The second one effectively modifies the value of the cavity detuning parameter $\theta$ according to the following relation:

$$
\theta_{K}=\theta+a K^{2}
$$

where $a$ being the diffraction parameter. For large values of $K$, this may eventually lead to a destabilization of traveling CS solution, as it happens in our case in Fig. 3. In order to check if this interpretation is correct, we numerically calculated the existence domain of CS in the parameter space $(\theta, K)$ using Eqs. (1) and (2). We have found that the limits of the CS existence domain, called $p$ and $q$ for $K=0$, change according to the law expressed by Eq. (4) as shown in Fig. 4.

We note that in our system, contrary to the case of the single equation model of Ref. [9], the change of variable $F$ $=E \exp (-i \vec{K} \cdot \vec{x})$ performed after introducing a phase gradient does not allow to conclude that any solution of the system without gradient will also exist, but move, in the presence of a gradient. In fact, Eqs. (1) and (2) are not preserved upon the addition of the phase gradient in the injected field, as shown by the absence of the convective term in the equation for the carriers. This absence could suggest that CS will loose stability for sufficiently high drift speed due to slow carrier dynamics. On the contrary, Fig. 4 clearly indicates that the mechanism for the saturation of CS velocity and their subsequent destabilization in presence of a phase gra-
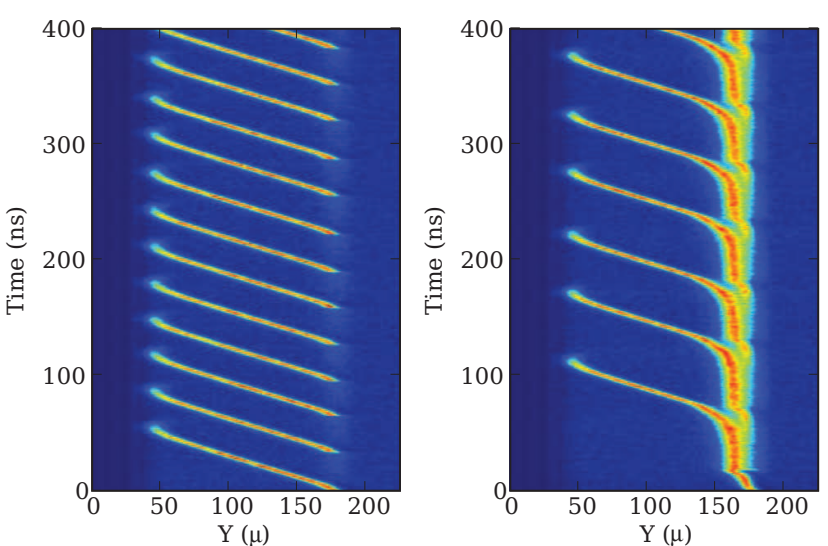

FIG. 5. (Color online) Spatiotemporal diagrams of the CS evolution [two-dimensional (2D) numerical simulation, cut along $x$ ] in the cases of shallow defect $\left(\theta_{d}=0.2\right.$, left panel $)$ and deep defect $\left(\theta_{d}=0.5\right.$, right panel). The defect is placed at $Y=180 \mu \mathrm{m}$, while in the rest of the sample the value of the detuning is given by $\theta_{0}$ $=-2$. Gradient direction is opposite to $Y$ axis. The intensity is represented in color scale increasing from blue to red. Other parameters are $K=0.052 \mu \mathrm{m}^{-1}, I=2$, and $E_{I}=0.8$.

dient in our system is, at least for the present parameter set, due to the correction to $\theta$ expressed by Eq. (4), directly implied by the presence of the phase gradient in the holding beam.

\section{DEFECTS AS SOURCES OF DRIFTING CS: THE CS TAP}

We consider a VCSEL device injected by a holding beam for parameters set where CSs exist in the largest part of the device section. We consider an attractive defect deep enough to lead to the formation of a structure, as described in Sec. II A. In these conditions, the application of a phase gradient in the holding beam phase may result in the formation of a periodic flow of drifting CSs originated in the defect. CS drift occurs in the direction of the gradient, thus climbing the slope of the phase. This spatiotemporal dynamics has been experimentally observed and theoretically analyzed recently [20]: if the force engendered by the phase gradient is strong enough to overcome the pinning force of the defect, the structure leaves the defect. Outside of the defect, both the CS and the low-intensity homogeneous solution are stable. Here, the structure becomes a CS and it keeps on traveling in the direction of the gradient with a speed depending on $K$. In the defect, once the structure has gone away, the system must relax back to the high-intensity ("pattern") state, which is the unique stable solution. Therefore, the structure forms deterministically in the defect as a result of the (local) evolution of the system to the equilibrium. This structure is pushed outside of the defect by the phase gradient and the process starts again, originating a periodic flow of drifting CS.

Numerical simulations of the spatiotemporal evolution of CS are represented by space-time diagrams in Fig. 5. In order to describe the observed dynamics, we introduce two characteristic times: the formation time $\left(\tau_{f}\right)$ of the structure in the defect after the preceding structure has gone and the detach- 


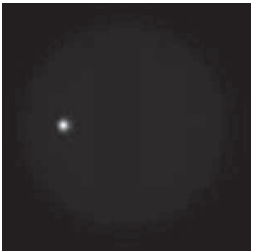

(a)

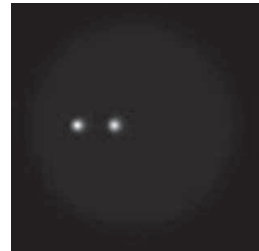

(b)

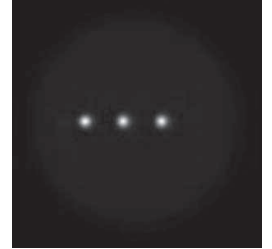

(c)

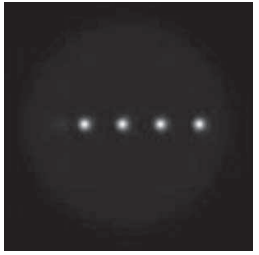

(d)

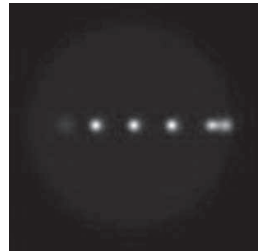

(e)

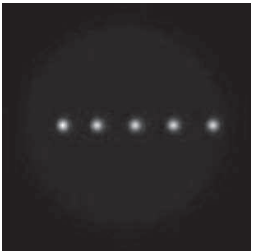

(f)

FIG. 6. Sequence of snapshots showing the spatiotemporal dynamics of drifting CSs in the transverse section of the VCSEL in presence of a shallow defect. Intensity increases from black to white. The position of the defect corresponds to the high-intensity structure visible in the first panel and the phase gradient is directed rightwards. Defect characteristics: $\theta_{0}=-2.0472, \theta_{d}=0.25$, and $\sigma_{d}=11.25 \mu \mathrm{m}$. Other parameters are $E_{I}=0.792, I=2.0024, K=0.052 \mu \mathrm{m}^{-1}$, and $t_{i}=5 \mathrm{~ns}$. The integration window measures about $280 \times 280 \mu \mathrm{m}$.

ing time $\left(\tau_{d}\right)$, i.e., the time during which a structure remains trapped in the defect after its formation. Two limit situations can be distinguished in terms of these times; both situations being characterized by a fully deterministic spatiotemporal evolution of CS. The first situation is characterized by no persistence of the structure inside the defect: once it is formed, it leaves immediately the defect $\left(\tau_{f} \gg \tau_{d}\right)$. This case (Fig. 5, left panel) is obtained for shallow defects and it has been experimentally evidenced in [20]. The second situation (Fig. 5, right panel) is characterized by a relatively long persistence of the structure into the defect $\tau_{d} \gg \tau_{f}$ and it is found when dealing with deep defects whose steep walls strongly pin the structure. The periodic flow of CS outside the defect is characterized by a period $T$ and a distance $\lambda$ between CS, $T$ corresponds to the time separation between the passages of two consecutive CSs on a point of the VCSEL transverse section. Then $\lambda / T=v, v$ being the speed of the CS. Let us analyze the spatiotemporal dynamics of CS in each situation.

\section{A. Shallow defect: $\tau_{f} \gg \tau_{d}$}

In Fig. 6, we display a sequence showing the startup of the periodic CS flow for the case of a shallow defect $\left(\theta_{d}\right.$ $=0.25$ ). In this situation, the period $T$ of the CS flow is $T$ $\approx \tau_{f}$ and $\tau_{f}$ turns out to be strongly dependent on system parameters and defect characteristics.

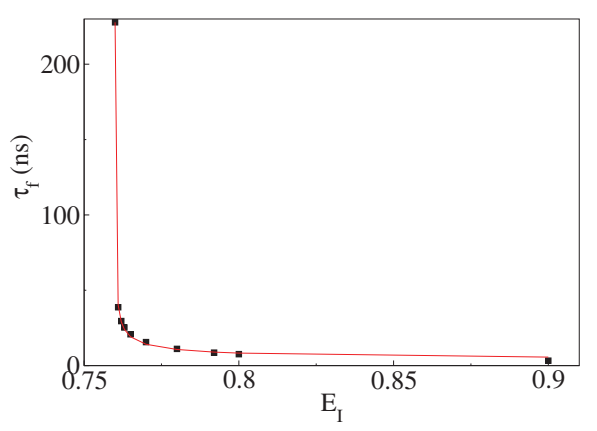

FIG. 7. (Color online) Structure formation time on a defect as a function of the holding beam amplitude $E_{I}$. At $t=0$, the holding beam amplitude is suddenly increased from the value $E_{I}=0.1$ to the new stationary value $E_{I}$. We plot here $\tau_{f}$ as a function of $E_{I}$. Other parameters values are $\theta_{0}=-2.0472, \theta_{d}=0.2, I=2.0024$, and $K$ $=0 \mu \mathrm{m}^{-1}$. The fitting function is $\tau_{f}=\tau_{0}+\left(E_{I}-E_{I, c}\right)^{b}: \tau_{0}=5.18 \mathrm{~ns}$, $E_{I, c}=0.79063$, and $b=-0.58$.
As previously explained, $\tau_{f}$ is related to the time required for the system to relax back to the stable solution (the structure) once the preceding one has gone. We have analyzed $\tau_{f}$ as a function of different bifurcation parameters of the system, namely, the value of $\theta$ in the defect bottom $\left(\theta_{0}-\theta_{d}\right)$, the input field amplitude $E_{I}$, and the VCSEL bias current $I$, in absence of the gradient. This dependence can be understood in terms of critical slowing down of a system close to a bifurcation. While there is no theoretical analysis of this phenomenon available in spatially extended bistable systems, critical slowing down has been widely investigated in zerodimensional systems [24]. In this case, the relaxation time is characterized by a dependence on the bifurcation parameter given by $\left|\mu-\mu_{c}\right|^{-1 / 2}$, with $\mu$ being the generic parameter and $\mu_{c}$ its critical value at the bifurcation [24]. As shown in Figs. 7 and 8, the fits of the numerical curves of $\tau_{f}$ reveal similar laws as a function of $E_{I}$ and $\theta_{0}-\theta_{d}$, though the scaling exponent differs from $\frac{1}{2}$.

It is worth pointing out again that the value $\theta_{c}=-2.114$ for which the formation time $\tau_{f}$ diverges in Fig. 8 is slightly different from $p=-2.06$, which is the corresponding value obtained for a perfectly homogeneous device (see Fig. 1). This is due to the limited size of the defect, as already discussed in Sec. II A.

As for the dependence of $\tau_{f}$ on the VCSEL bias, it appears that changing this parameter does not correspond only to change the parameter $I$ in the equations. A change in the

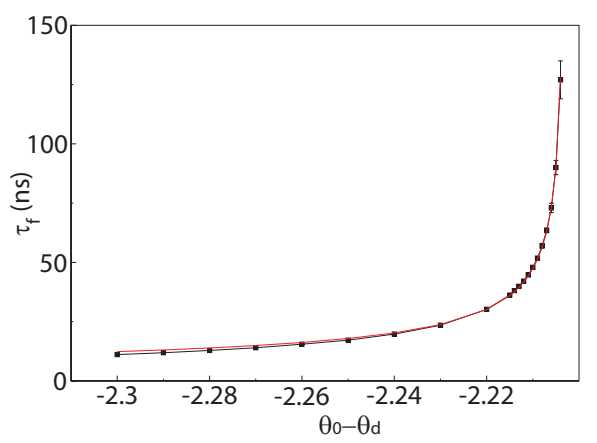

FIG. 8. (Color online) Structure formation time $\tau_{f}$ as a function of the value of $\theta$ at the defect bottom $\theta_{0}-\theta_{d}$. The defect profile is introduced in the numerical simulations at $t=0$, starting from the stationary solution corresponding to the perfectly homogeneous case. Other parameters are $\theta_{0}=-2.0472, I=2.0024, E_{I}=0.792$, and $K=0 \mu \mathrm{m}^{-1}$. The fitting functions are $\tau_{f}=\tau_{0}+\left(\theta_{c}-\theta\right)^{b}, \tau_{0}=7.27 \mathrm{~ns}$, $b=-0.76$, and $\theta_{c}=-2.114$. 


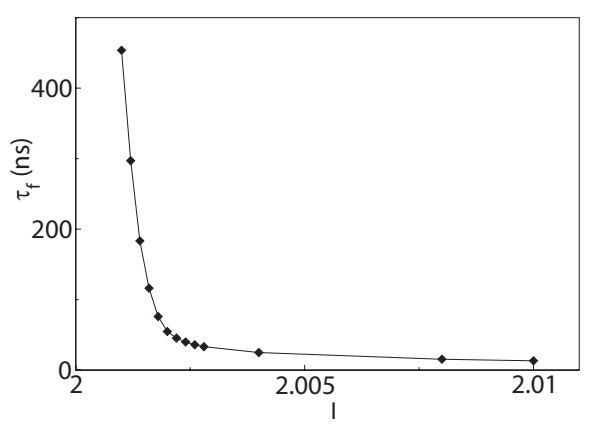

FIG. 9. Structure formation time $\tau_{f}$ as a function of VCSEL bias. The defect profile is introduced in the numerical simulations at $t=0$, starting from the stationary solution corresponding to the perfectly homogeneous case. The defect depth is $\theta_{d}=0.2$, while $\theta_{0}$ changes with the injected current due to Joule heating (see text). Other parameters are $K=0 \mu \mathrm{m}^{-1}$ and $E_{I}=0.792$.

VCSEL bias implies also a variation of $\theta_{0}$ because of the change of the semiconductor refractive index caused by Joule heating. In order to take into account this double effect in the model, we have phenomenologically introduced a linear dependence of $\theta_{0}$ on $I$ [25] by putting $\theta_{0}=\overline{\theta_{0}}-5(I-\bar{I})$, being $\overline{\theta_{0}}=-2.0472$ and $\bar{I}=2.0024$ [14]. The dependence of $\tau_{f}$ as a function of VCSEL bias is plotted in Fig. 9.

Outside of the defect, the CS flow is characterized by their drifting speed $v$ which depends on the holding beam phase gradient $K$, according to Fig. 3. The influence of $K$ does not concern only $v$ but also $\tau_{f}$ due to the effective correction to the detuning value of the whole device given by Eq. (4) engendered by the application of the phase gradient, as explained in Sec. II B. Therefore, also the value of $\theta$ at the defect bottom is affected by this correction and, as a consequence, $\tau_{f}$ increases as well as shown in Fig. 10 . When $K$ exceeds a critical value, the structure formation does not occur anymore on the defect $\left(\tau_{f} \rightarrow \infty\right)$. This explains why the curve in Fig. 10 stops for $K$ larger than $0.055 \mu \mathrm{m}^{-1}$.

The separation $\lambda$ between drifting CSs is given by $\lambda$ $=v \tau_{f}$. This is a very important characteristic of the spatiotemporal dynamics observed because if the CSs get too close each other, they interact. This interaction leads to a complex behavior and destroys the regular flow of CS, as it will be shown in Sec. III C. The dependence of $\lambda$ on $K$ is plotted in Fig. 10. Since both $\tau_{f}$ and $v$ increase with $K$, also the distance between drifting CSs increases with $K$, which is beneficial in order to avoid interactions. This is particularly im-

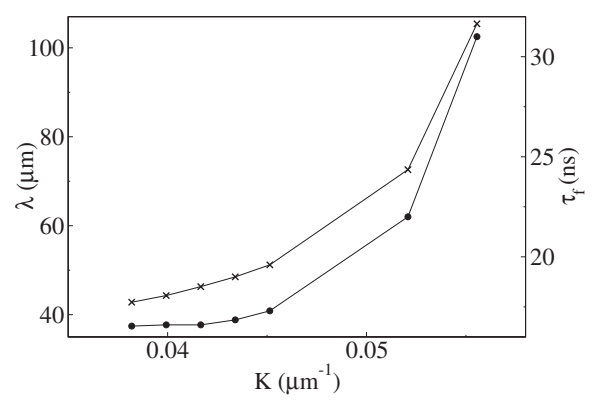

FIG. 10. Structure formation time in the defect $\tau_{f}$ (circles) and distance $\lambda$ (crosses) between drifting CSs measured outside the defect $(80 \mu \mathrm{m}$ far apart) as a function of $K$ in presence of a shallow defect $\theta_{d}=0.20$. All other parameters are as in Fig. 6

portant for applications such as the delay line or the shift register [15]. As a matter of fact, on the left side of Fig. 10, the curve stops because for smaller values of $K$, no regular periodic regime is observed anymore: the distance between two successive drifting CSs becomes smaller than their interaction distance (see also Sec. III C).

\section{B. Deep defect: $\tau_{d} \gg \tau_{f}$}

The second regime that we will analyze is the one observed in presence of a deep defect. It is characterized by the persistence of the structure inside the defect after its regeneration, as shown in the spatiotemporal diagram of Fig. 5, right panel. In Fig. 11, we display a sequence showing the startup of the periodic CS flow for the case of a deep defect $\left(\theta_{d}=0.45\right)$.

In this case, the steepness of the defect walls tightly pins the structure and the value of the phase gradient applied becomes critical in order to detach the structure. If the force engendered by the holding beam phase gradient is barely stronger than the pinning force of the defect, then the structure velocity is very small close to the defect walls and it takes a long time to escape from the defect. While in the case of a shallow defect $\tau_{d}$ is negligible with respect to $\tau_{f}$, the opposite happens in the case of a deep defect and the period of the CS flow is $T \approx \tau_{d}$. As a matter of fact, $\tau_{f}$ becomes smaller as the defect gets deeper (i.e., as $\theta_{d}$ increases, see Fig. 8), thus the dynamics is ruled by $\tau_{d}$. At difference from $\tau_{f}, \tau_{d}$ is not affected by the system parameters $E_{I}$ and $I$. Instead, given a defect depth, $\tau_{d}$ depends critically on $K$ and it decreases when $K$ is increased, since the speed of the struc-

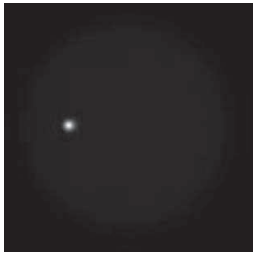

(a)

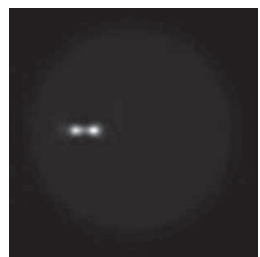

(b)

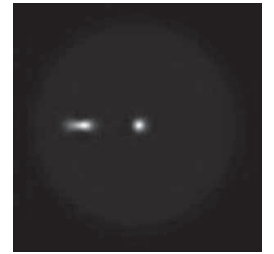

(c)

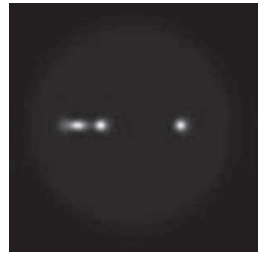

(d)

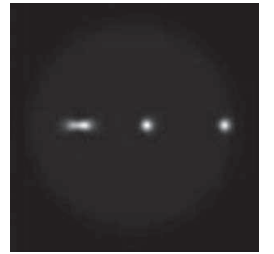

(e)

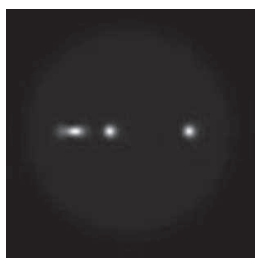

(f)

FIG. 11. Sequence of snapshots showing the spatiotemporal dynamics of drifting CSs in the transverse section of the VCSEL in the presence of a deep defect. The position of the defect corresponds to the high-intensity structure visible in the first panel and the phase gradient is directed rightwards. The defect characteristics are as in Fig. 6 except for $\theta_{d}=0.45$. Other parameters are as in Fig. 6. 


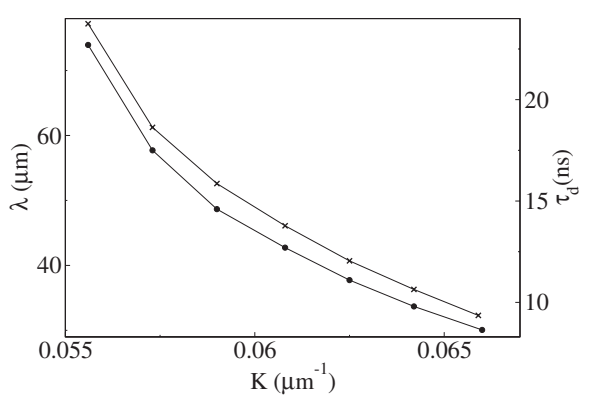

FIG. 12. Structure detaching time in the defect $\tau_{d}$ (circles) and distance $\lambda$ (crosses) between drifting CSs measured outside the defect $(80 \mu \mathrm{m}$ far apart) as a function of $K$ in presence of a deep defect: $\theta_{d}=0.5$. All other parameters are as in Fig. 6 .

ture inside the defect is increased. In an equivalent manner, for a fixed phase gradient, $\tau_{d}$ is strongly dependent on the defect depth and steepness since, as shown in Fig. 2, the deeper is the defect, the stronger is its pinning force.

The dependence of $\tau_{d}$ and of the separation between drifting CSs $(\lambda)$ is plotted in Fig. 12 as a function of $K$. On the left side of the figure, the curves stop when $K$ is too small to detach the structure from the defect $\left(\tau_{d} \rightarrow \infty\right.$ and $\left.\lambda \rightarrow \infty\right)$. On the right side, for large value of $K$, both $\tau_{d}$ and $\lambda$ become too small and no regular periodic regime is observed because of CS interactions (see also Sec. III C). By further increasing $K$, the value of $\theta$ at the defect bottom increases due to the correction (4). Thus, $\tau_{f}$ increases accordingly and it eventually becomes larger than $\tau_{d}$, thus leading to the situation described in Sec. III A. Further increasing of $K$ affects CS stability outside the defect, as described in Sec. II B.

\section{Overview and hybrid situation}

As a function of the defect depth $\theta_{d}$ and the gradient strength $K$ in the holding beam phase, the whole set of different spatiotemporal regimes has been mapped in Fig. 13. Region I is the one characterized by $\tau_{f} \gg \tau_{d}$ (shallow defects), while region II is the one where $\tau_{f} \ll \tau_{d}$ (deep defects). Hybrid situations characterized by strong interactions between the drifting CSs appear in between (region III). In this regime, complex cooperative effects as bunching and collapsing are observed, as well as the formation of a propagative filament, as shown in Fig. 14. In region IV, no dynamics is observed because $\tau_{d}$ is infinite: the structure cannot leave the defect because the pinning force is larger than the force

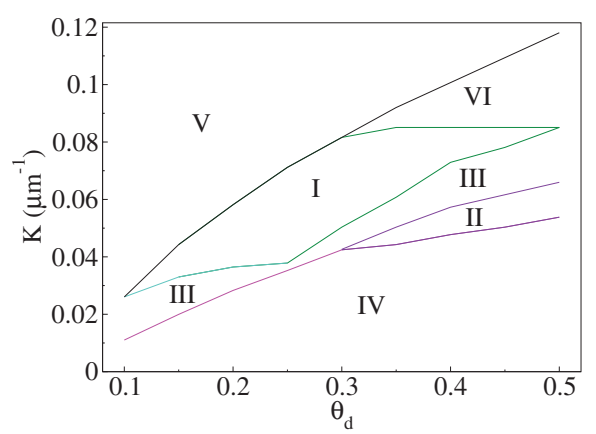

FIG. 13. (Color online) Qualitative map obtained by observing numerically the different spatiotemporal regimes as a function of $\theta_{d}$ and $K$. All other parameters are as in Fig. 6

engendered by the phase gradient. As expected, the critical value of $K$ necessary to overcome the pinning force increases with the defect depth. In region $\mathrm{V}$, no spontaneous formation of the structures is observed in the defect because the value of $\theta$ at the bottom of the defect becomes larger than the critical value above which $\tau_{f}$ goes to infinite. Finally, region VI corresponds to the situation where no dynamics is observed because the CS is unstable outside the defect, though $\tau_{f}$ remains finite and a structure spontaneously forms in the defect. This is due to the correction (4), as already mentioned in Sec. II B. In this case, as soon as the structure is detached, it dies after a short propagation.

\section{COLLISION BETWEEN A DEFECT AND A MOVING CS}

In this section, we analyze the case of a CS drifting in the transverse section of the device when it collides with a defect. CS movement may be perturbed by defects since the defect itself engenders a parameter gradient $(\theta$ in our description) that entails a force on the drifting CSs [22]. This force competes with the one exerted by the holding beam phase gradient, resulting in a trajectory that may deviate from the direction fixed by the phase gradient [19]. CS trajectory may be forced along the phase gradient direction by injecting the holding beam in form of a stripe with the same direction of the phase gradient. The intensity profile of the holding beam creates a channel along which the CS is bounded [15]. In this case, the trajectory of the CS cannot be deviated from the direction fixed by the channel, but the value of CS speed is affected by the presence of the defect

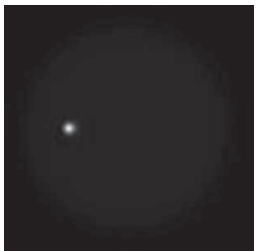

(a)

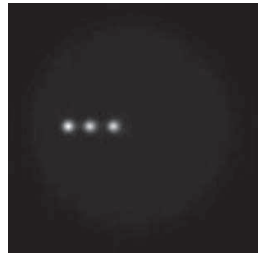

(b)

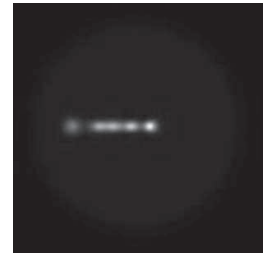

(c)

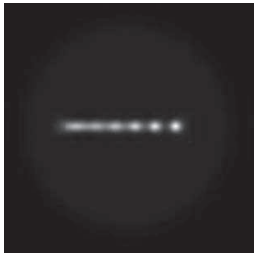

(d)

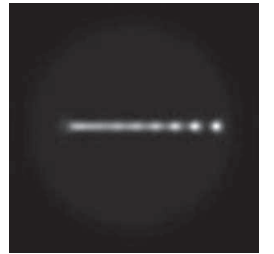

(e)

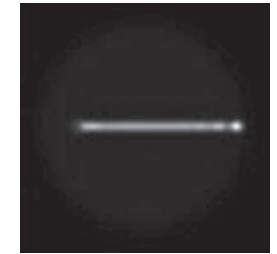

(f)

FIG. 14. Sequence of snapshots showing the spatiotemporal dynamics of drifting CSs in the transverse section of the VCSEL. The position of the defect corresponds to the high-intensity structure visible in the first panel and the phase gradient is directed rightwards. The defect characteristics are as in Fig. 6 except for $\theta_{d}=0.35$. All other parameters are as in Fig. 6. 


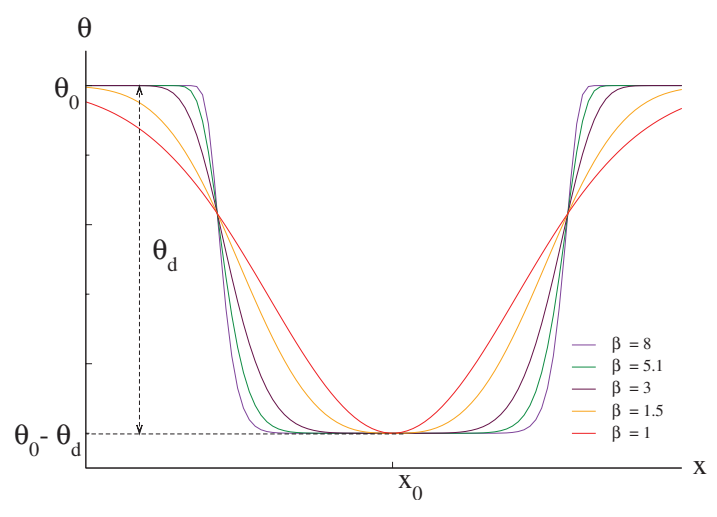

FIG. 15. (Color online) Super-Gaussian attractive defect profile (cut at $y=y_{0}$ ) for different values of $\beta$. Other defect characteristics are $\theta_{d}= \pm 0.03, \theta_{0}=-2.0$, and $\sigma_{d}=27 \mu \mathrm{m}$.

and CS may be pinned by the defect walls. We analyze this situation, which has been experimentally reported in [15], in presence of both attractive and repulsive defects.

In the perturbative limit (small gradients), the CS speed at a point of the space is proportional to the value of the parameter gradient in this point [21]. Therefore, the key element defining the interaction between the defect and the moving CS is the steepness of the defect walls, i.e., the derivative of $\theta$, calculated in the direction of the motion (that we identify with the $x$ axis). The comparison between the force engendered by the holding phase gradient and the pinning force of a defect can be realized by analyzing the CS speed when crossing a defect for different defect steepness. This can be varied by changing the value of $\beta$ in Eq. (3), but maintaining the same values for $\theta_{0}$ and $\theta_{d}$, as shown in Fig. 15. The defect width $\sigma_{d}$ has been enlarged with respect to the one used in Sec. III in order to isolate the pinning effect of a single defect wall on the drifting CS. Otherwise, the finite size of CS makes unavoidable that the edge part of CS interacts with the second wall while the back part is still interacting with the first wall. Furthermore, the value of $\theta_{d}$ is chosen such that the value of $\theta$ at the defect bottom or top is compatible with CS existence. Then, the moving CS will survive when crossing the defect and no spontaneous generation of structures occurs on the defect.

The result of our analysis is plotted in Fig. 16 where, together with the defect profile and its derivative, we plot the CS speed as a function of the spatial position. Here, the phase gradient is directed rightwards and the CS moves from left to right. The plotted curves indicate that the CS speed varies significantly when interacting with defect walls. In the case of a moderately steep attractive defect [Fig. 16(a)], the CS undergoes an acceleration upon entering the defect (left boundary) and is decelerated when exiting the defect (right boundary), i.e., when the effects of phase and detuning gradients tend to compensate each other.

When the steepness of the defect becomes sufficiently large, the velocity of the CS at the right edge of the defect will eventually vanish, causing a pinning of the CS. We remark that as soon as the defect steepness overcomes the minimal value required to compensate the effect of the phase gradient, not only one but two points with zero velocity (i.e.,
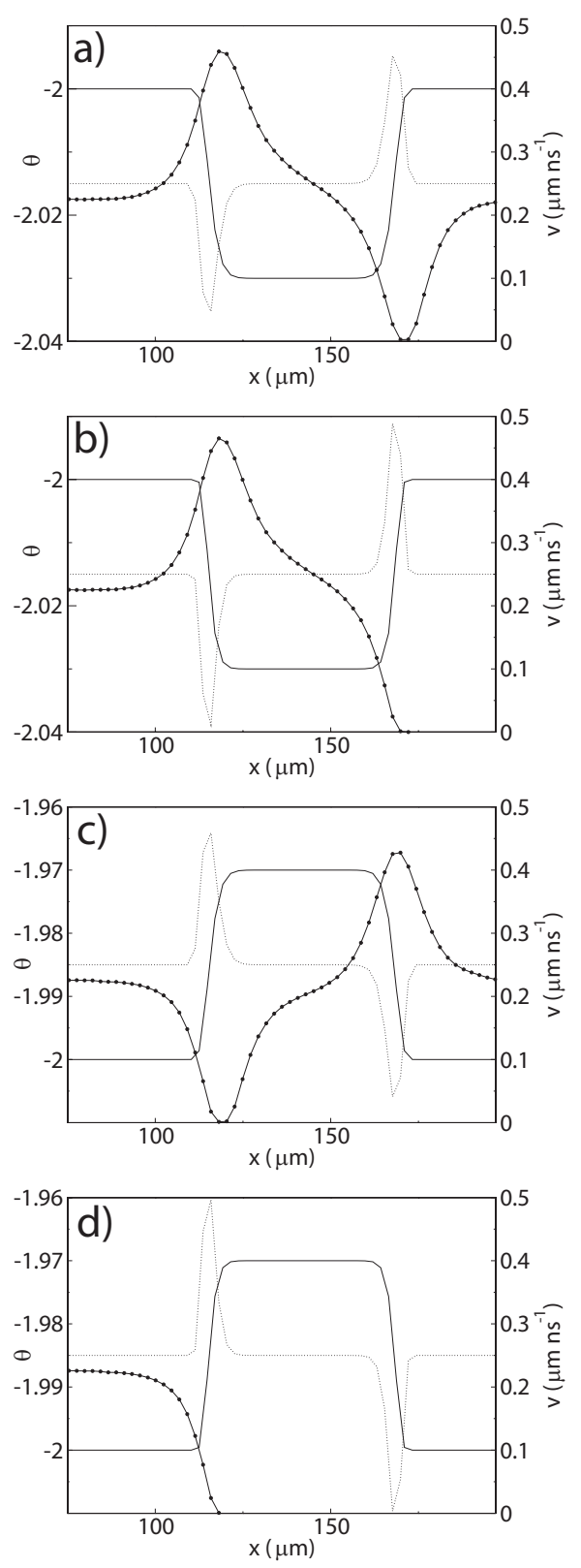

FIG. 16. CS speed when crossing an [(a) and (b)] attractive-[(c) and (d)] repulsive defect. The holding beam phase gradient has the same direction of $x$ axis. The CS speed is plotted by the black line with points while the defect profile is plotted by the gray line. The dotted line is the derivative of $\theta: d \theta / d x$. The defect wall steepness, i.e., the value of $d \theta / d x$, is changed without changing the defect depth or height which is fixed to $\theta_{d}= \pm 0.03$ with $\theta_{0}=-2.0$ and $\sigma_{d}=27 \mu \mathrm{m}$. We change the value of the exponent $\beta$ in Eq. (3). In (a) and (c), $\beta=7$ and the maximum value of the derivative of $\theta$ (dotted line) is $0.005 \mu \mathrm{m}^{-1}$. In (b) and (d), $\beta=9$ and the maximum value of the derivative of $\theta$ (dotted line) is $0.006 \mu \mathrm{m}^{-1}$. Other parameters are $K=0.0038 \mu \mathrm{m}^{-1}, E_{I}=0.792$, and $I=2.0$.

fixed points) will exist. These two fixed points, spatially separated by a region of negative (leftwards) velocity, will have opposite stabilities: the left one being stable and the right one being unstable. On Fig. 16(b), a CS coming from the left side of the device is stuck on the stable fixed point 
and will therefore never exit the defect. It is interesting to note that the transition from the pinning to unpinning of a CS on a defect, involving the collision and disappearance of two fixed points, corresponds to a saddle-node bifurcation which takes place for a defect steepness such that the maximum of the detuning gradient exactly compensates the phase gradient.

A similar phenomenon occurs in the case of a repulsive defect [Figs. 16(c) and 16(d)] with the CS velocity increasing when the effects of phase and detuning gradients add up (right side of the defect in this case) and velocity decreasing eventually down to zero when they compensate [left side of the defect, Fig. 16(d)]. It is important to notice that, in absence of the intensity channel which reduces the motion essentially to one dimension (1D), in the case of the repulsive defect, the traveling CS would be free to avoid the defect, deviating from its trajectory, instead of being pinned at the defect feet.

\section{CS APPLICATIONS IN PRESENCE OF DEVICE DEFECTS}

Some of the most promising applications of CS rely on their property to drift in the presence of a parameter gradient. The easiest form to implement an external and controllable gradient is to introduce a phase gradient in the holding beam. The movement of CS under this gradient can be exploited for all-optical delay lines and shift-register memories and it has been experimentally demonstrated in a semiconductor microresonator in [15]. One of the outcomes of these experiments was that defects of the semiconductor device may affect detrimentally these applications. Hence, it is very important to characterize their interaction with holding beam phase gradients introduced for propelling CS along the device section. This comparison requires numerical integration of system equations for different defect steepness and for different gradient strength, since it is not straightforward to compare the forces engendered by different parameters gradients. In Sec. IV, we have shown numerically how the defect interacts with a CS propelled by a gradient in the phase of the holding beam. We have also calculated the critical value of the defect steepness above which the CS drift is stopped. This analysis confirms also that the CS speed is, at each point of the space, linked to the value of the gradient of the defect profile, thus indicating that the steepness of defect walls is the key parameter in the interaction between the defect and the drifting CS.

In this paper, we have also shown that the interplay between device defects and a phase gradient in the holding beam may lead to a regular sequence of CSs springing from the defect and moving in the gradient direction, which we called CS tap. Our study suggests that defectlike spots can be engineered in the transverse plane of the device in order to create device built-in CS sources, thus avoiding the use of a writing beam to generate CS.

CS generation rate can be set by controlling the system parameters or by engineering the defect characteristics. Defects with different height or depth and size could be engineered to obtain simultaneously CS sources with different

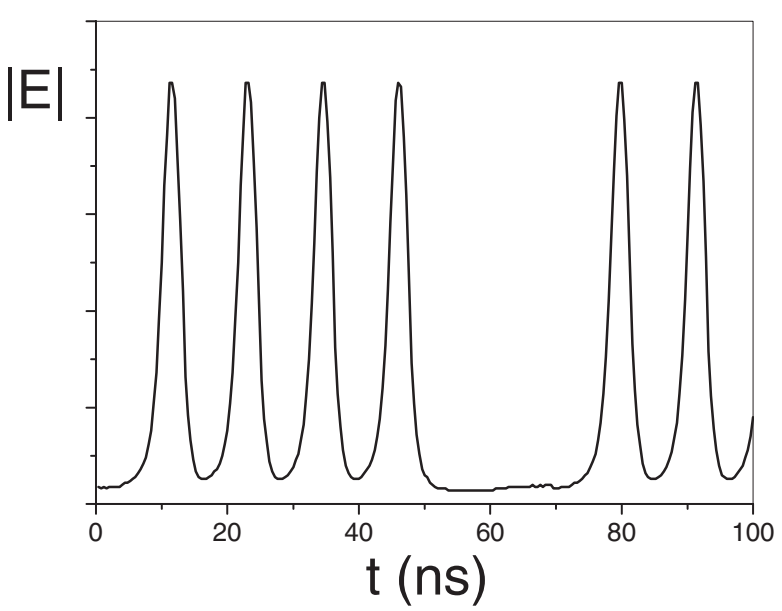

FIG. 17. Time trace in a point on the "channel" defined by the gradient direction, situated $22.5 \mu \mathrm{m}$ apart from the defect, when a narrow incoherent beam is injected into the defect stopping the CS production. As soon as the injection stops, the CS flux starts again. The duration of the simulation is $100 \mathrm{~ns}$, with $50 \mathrm{~ns}$ free evolution, $10 \mathrm{~ns}$ injection inhibition, and $40 \mathrm{~ns}$ free evolution. Parameters are $\theta_{d}=0.2, \theta_{0}=-2.0, I=2, E_{I}=0.8$, and $K=0.042 \mu \mathrm{m}^{-1}$.

properties in the same device. The behavior upon variations of the VCSEL bias current suggests that, by means of a weak modulation of the pumping current, it may be possible to modulate the distance between CS and the velocity of the flow, keeping constant the value of the phase gradient generating the motion. Moreover, it may be even possible to stop the CS generation without perturbing either the CS stability or their speed outside the defect by simply varying the pumping level across the critical value where the formation time of the structure goes to infinity (see Fig. 9).

This sensitivity of CS generation on the pumping current also suggests the possibility of realizing a delay line for digital signals where a bit value of " 1 " is stored as a drifting CS and the input signal is used for modulating the VCSEL pumping current. This represents an optoelectronic method to store information bits in form of moving CS, thus realizing a serial to parallel converter. For applications such as the delay line, it is important that no "errors" appear during the spatial propagation: the spatial separation among the different pulses must be such that no interactions take place among the moving CS. A deep knowledge of the spatiotemporal behavior of the system as a function of the key parameters (typically, $K$ and $\theta$ ), such as the one summarized in Fig. 13 would allow for a correct working of the line. In particular, it will be important to avoid the region labeled as III, where the drifting CS may interact along the line affecting the data series to transmit. Studying curves such as those shown in Figs. 10 and 12 would be of great help to realize such devices.

Finally, the CS flow can also be modified by injecting an optical perturbation onto the defect originating the flow. In particular, it is possible to interrupt the CS flux for a certain interval of time by injecting a Gaussian incoherent beam in the defect position without varying all the other parameters. This acts as an erasing beam and, as long as it is on, it stops the CS emission. When the injected beam is switched off 

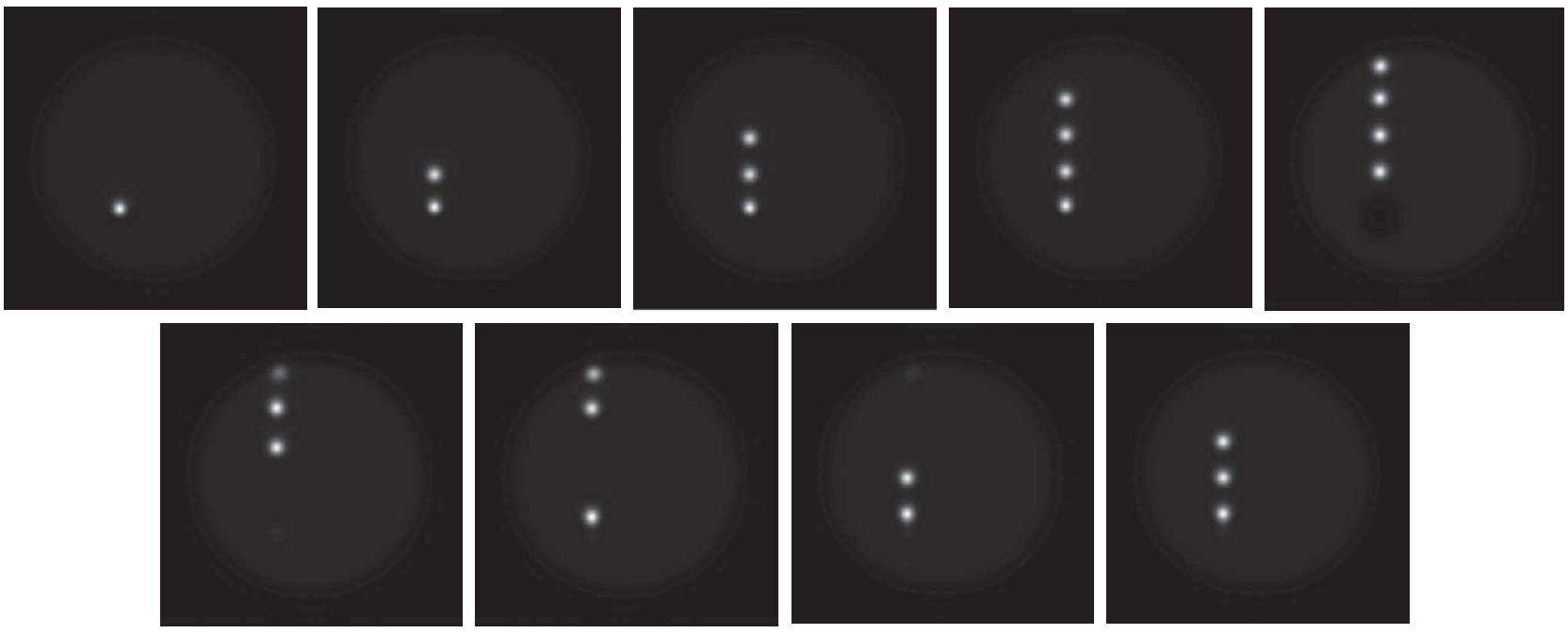

FIG. 18. Sequence of snapshots illustrating different stages of continuous creation and inhibition of CSs (same simulation as Fig. 17). The defect position corresponds to the CS in the first frame and the phase gradient is directed upwards. The injection starts just before frame 5 and stops after frame 6. From left to right, the frames are taken every $12 \mathrm{~ns}$, starting at the fourth ns $(4,16,28,40,52,64,76,88,100 \mathrm{~ns})$.

again, a new structure is spontaneously created in the defect and the CS flux starts again. We obtain therefore a perfect control on CS emission and this would permit us to realize a delay line where information is encoded by erasing pulses in a periodical sequence instead of writing them. At difference with the use of a writing beam, this operation can be realized by using an optical perturbation not necessarily coherent with the holding beam, thus simplifying remarkably the experimental scheme. In Figs. 17 and 18, we show a numerical simulation where we implement this operation. After emission of four drifting CS, we inject on the defect an incoherent narrow beam for $10 \mathrm{~ns}$ and we are able to suppress CS emission. When the perturbation is removed, the CS emission starts again. In Fig. 17, we show the time trace in a point $22.5 \mu \mathrm{m}$ apart from the defect along the channel defined by the gradient direction. The sequence of pulse results inhibited during the injection (from $T=50 \mathrm{~ns}$ to $T=60 \mathrm{~ns}$ ) and does not start again until a new structure is created at the defect position and removed by the gradient. In Fig. 18, we show a sequence of snapshots illustrating different stages of continuous creation and inhibition of CSs.

\section{CONCLUSIONS}

In this paper, we have analyzed numerically the effects of device defects on drifting CS. Device defects have been modeled as small-scale variations of the cavity detuning parameter.

We have analyzed the spatiotemporal dynamics arising from the interaction between defects and a holding beam phase gradient, shedding light on the periodic regime of CS springing from the defect that has been recently experimentally observed [20]. We have identified two regimes, where the CS flow is ruled by two different characteristic times: the time required for the structure formation upon the defect and the detaching time, i.e., the time for the structure to leave the defect. While the former is strongly influenced by system parameters, the latter depends mainly on the gradient strength in the holding beam phase. We have mapped the different spatiotemporal regimes in the parameter space $\left(K, \theta_{d}\right)$ where the two regimes described before can be individuated. Other regimes are possible, where the CS flow becomes irregular due to interactions among the moving CS.

We have also shown that the introduction of the phase gradient of the holding beam has several consequences on the dynamics of drifting CS. Besides pushing the structure out of the defect and determining the CS speed outside of the defect, the phase gradient implies an effective correction to the cavity detuning parameter of the device, which may influence CS stability. We have shown how this correction, for large phase gradient, affects deeply the CS spatiotemporal dynamics.

We have also studied the collision between a traveling CS and a defect placed on its trajectory, which may affect detrimentally applications such as CS-based delay lines. We have compared the force engendered by the phase gradient inducing the motion and the pinning force generated by the defect. Whether CS stops or passes through the defect depends on which of these two forces is stronger.

Finally, we proposed some applications based on the periodic generation of traveling CS. In particular, we have demonstrated numerically that the CS flow can be stopped by injecting a narrow incoherent pulse onto the defect. This operation allows for external control of the CS flow and thus for the incoherent encoding of information along a delay line based on this periodic regime.

\section{ACKNOWLEDGMENTS}

We thank Lionel Gil for useful discussions. G.T. acknowledges the financial support of the University of Nice SophiaAntipolis. 
[1] F. Wise and P. Di Trapani, Opt. Photonics News 13, 28 (2002).

[2] Dissipative Solitons, edited by N. Akhmediev and A. Ankiewicz, Lecture Notes in Physics Vol. 661 (Springer, New York, 2005), Chaps. 3-6.

[3] N. N. Rosanov, Spatial Hysteresis and Optical Patterns (Springer, New York, 2002).

[4] O. Thual and S. Fauve, J. Phys. (Paris) 49, 1829 (1988).

[5] L. A. Lugiato, IEEE J. Quantum Electron. 39, 193 (2003).

[6] N. Rosanov and G. Khodova, Opt. Spectrosc. 65, 449 (1988).

[7] M. Tlidi, P. Mandel, and R. Lefever, Phys. Rev. Lett. 73, 640 (1994).

[8] M. Brambilla, L. A. Lugiato, and M. Stefani, Europhys. Lett. 34, 109 (1996).

[9] W. J. Firth and A. J. Scroggie, Phys. Rev. Lett. 76, 1623 (1996).

[10] M. Brambilla, L. A. Lugiato, F. Prati, L. Spinelli, and W. J. Firth, Phys. Rev. Lett. 79, 2042 (1997).

[11] L. Spinelli, G. Tissoni, M. Brambilla, F. Prati, and L. A. Lugiato, Phys. Rev. A 58, 2542 (1998).

[12] S. Barland, J. R. Tredicce, M. Brambilla, L. A. Lugiato, S. Balle, M. Giudici, T. Maggipinto, L. Spinelli, G. Tissoni, T. Knoedl, M. Miller, and R. Jaeger, Nature (London) 419, 699 (2002).

[13] X. Hachair, S. Barland, L. Furfaro, M. Giudici, S. Balle, J. R. Tredicce, M. Brambilla, T. Maggipinto, I. M. Perrini, G. Tissoni, and L. A. Lugiato, Phys. Rev. A 69, 043817 (2004).

[14] X. Hachair, L. Furfaro, J. Javaloyes, M. Giudici, S. Balle, J. R.
Tredicce, G. Tissoni, L. A. Lugiato, M. Brambilla, and T. Maggipinto, Phys. Rev. A 72, 013815 (2005).

[15] F. Pedaci, S. Barland, E. Caboche, P. Genevet, M. Giudici, J. Tredicce, T. Ackemann, A. Scroggie, G. L. Oppo, W. J. Firth, G. Tissoni, and R. Jaeger, Appl. Phys. Lett. 92, 011101 (2008).

[16] F. Pedaci, P. Genevet, S. Barland, M. Giudici, and J. Tredicce, Appl. Phys. Lett. 89, 221111 (2006).

[17] J. Oudar et al., Opt. Quantum Electron. 24, S193 (1992).

[18] R. Kuszelewicz, I. Ganne, I. Sagnes, G. Slekys, and M. Brambilla, Phys. Rev. Lett. 84, 6006 (2000).

[19] F. Pedaci, G. Tissoni, S. Barland, M. Giudici, and J. Tredicce, Appl. Phys. Lett. 93, 111104 (2008).

[20] E. Caboche, F. Pedaci, P. Genevet, S. Barland, M. Giudici, J. Tredicce, G. Tissoni, and L. A. Lugiato, Phys. Rev. Lett. 102, 163901 (2009).

[21] T. Maggipinto, M. Brambilla, G. K. Harkness, and W. J. Firth, Phys. Rev. E 62, 8726 (2000).

[22] S. Fedorov, D. Michaelis, U. Peschel, C. Etrich, D. V. Skryabin, N. Rosanov, and F. Lederer, Phys. Rev. E 64, 036610 (2001).

[23] N. N. Rozanov, V. E. Semenov, and G. V. Khodova, Sov. J. Quantum Electron. 12, 193 (1982).

[24] S. Cribier, E. Giacobino, and G. Grynberg, Opt. Commun. 47, 170 (1983); F. Mitschke et al., ibid. 46, 135 (1983).

[25] C.-L. Tsai et al., IEEE Trans. Electron Devices 52, 1033 (2005). 